\title{
Crescimento de nogueira-pecã sob diferentes preparos do solo e coveamentos: coleópteros como bioindicadores
}

\author{
Jardel Boscardin*; Ervandil Corrêa Costa²; Eloi Paulus²; Dayanna do Nascimento Machado²; Leandra Pedron²; Pedro Giovâni da Silva ${ }^{3}$ \\ ${ }_{1}^{1}$ Universidade Federal de Uberlândia, Rod. LMG 746, km 1, Araras, CEP 38500-000, Monte Carmelo, MG, Brasil \\ ${ }^{2}$ Universidade Federal de Santa Maria, Av. Roraima, n. 1000, Camobi, CEP 97105-900, Santa Maria, RS, Brasil \\ ${ }^{3}$ Universidade Federal de Minas Gerais, Av. Presidente Antônio Carlos, 6627, Pampulha, CEP 31270-901, Belo Horizonte, MG, Brasil
}

*Autor correspondente:

jardel.boscardin@ufu.br

Termos para indexação:

Carya illinoinensis

Entomologia

Fauna do solo

Index terms:

Carya illinoinensis

Entomology

Soil fauna

Histórico do artigo:

Recebido em 12/09/2017

Aprovado em 11/12/2017

Publicado em 29/12/2017

doi: 10.4336/2017.pfb.37.92.1496
Resumo - O trabalho objetivou avaliar os efeitos de diferentes preparos do solo e coveamentos na implantação de um pomar de nogueira-pecã Carya illinoinensis (Wangenh.) K. Koch (Juglandaceae), através da fauna da Ordem Coleoptera. O pomar de nogueira-pecã foi implantado em fevereiro de 2012, em espaçamento de $7 \mathrm{~m} \mathrm{x}$ $7 \mathrm{~m}$, em Santa Maria, RS, em delineamento de blocos ao acaso, com cinco blocos e quatro tratamentos, compostos por plantio: em cova pequena de $20 \mathrm{~cm} \times 60 \mathrm{~cm}(\mathrm{Cp})$; subsolador mais grade niveladora e cova pequena (Scp); enxada rotativa e cova pequena (Ecp); e em cova grande de $40 \mathrm{~cm}$ x $60 \mathrm{~cm}(\mathrm{Cg})$. De março de 2014 a novembro de 2015 , foram realizadas oito coletas da fauna de Coleoptera, uma por estação do ano, com quatro armadilhas pitfall distribuídas em cada tratamento e cinco em cada área do entorno, totalizando 100 amostras por coleta. O tratamento Ecp apresentou os menores valores de diversidade, riqueza e equitabilidade para a coleopterofauna, enquanto que os tratamentos Cg e Cp apresentaram os maiores índices. A espécie Diloboderus abderus (Sturm) apresentou-se como muito frequente. Conclui-se que o tratamento $\mathrm{Cg}$ não interfere na diversidade de coleópteros e promove o maior crescimento em diâmetro de plantas de nogueira-pecã.

\section{Pecan growth under different soil preparation and hole sizes: coleopterans as bioindicators}

\begin{abstract}
The study aimed to evaluate the effects of different soil preparation and hole sizes in an orchard of Carya illinoinensis pecan (Wangenh.) K. Koch (Juglandaceae) by the Order Coleoptera fauna. A pecan orchard was established in spacing $7 \mathrm{~m} \times 7 \mathrm{~m}$, in Santa Maria, Rio Grande do Sul State, Brazil in randomized block design, with five blocks and four treatments, using: small pit $20 \mathrm{~m} \mathrm{x} 60 \mathrm{~cm}(\mathrm{Cp})$; more harrowing subsoiler and small pit (Scp); rotary hoe and small pit (Ecp); and large pit $40 \mathrm{~m}$ x $60 \mathrm{~cm}(\mathrm{Cg})$. From March 2014 to November 2015, eight samples were taken from Coleoptera fauna, one per season, with four pitfall traps distributed in each treatment and the surrounding area, totaling 100 samples per collection. The Ecp treatment had the lowest diversity values and evenness for Coleoptera fauna, while the $\mathrm{Cp}$ and $\mathrm{Cg}$ treatments presented the highest indices. The species Diloboderus abderus (Sturm) was as very common. It was concluded that $\mathrm{Cg}$ treatment does not interfere with coleopteran and promotes the highest growth in diameter of pecan plants.
\end{abstract}




\section{Introdução}

A nogueira-pecã Carya illinoinensis (Wangenh.) K. Koch pertence à família Juglandaceae e é originária dos Estados Unidos e México (Wells, 2013). Está presente no Brasil há mais de cem anos e vem ganhando espaço no sul do país, onde as áreas cultivadas estão em expansão, devido à alta demanda e ao consequente bom preço pago pelas nozes.

$\mathrm{Na}$ implantação do pomar de nogueira-pecã, entre os fatores determinantes para o bom desenvolvimento das plantas está o correto preparo do solo. Nesse aspecto, o tamanho das raízes das mudas no momento do transplante deve ser levado em consideração para a escolha da profundidade das covas, e o tipo de solo irá determinar o melhor preparo a ser empregado. Raseira (1990) propõe covas para transplante das mudas com $60 \mathrm{~cm}$ a $70 \mathrm{~cm}$ de profundidade e $50 \mathrm{~cm}$ de diâmetro. Fronza et al. (2013) sugerem a realização de lavração, gradagem e subsolagem, para um melhor estabelecimento do sistema radicular, sendo que as mudas devem ser plantadas em covas de $40 \mathrm{~cm}$ x $40 \mathrm{~cm}$ x $40 \mathrm{~cm}$.

O preparo do solo pode causar impacto na fauna edáfica, e entre as práticas que podem prejudicar de maneira drástica a estabilidade da fauna do solo, estão a aração e a gradagem. Essas práticas modificam a estrutura do solo e reduzem sua cobertura, intensificando o efeito da radiação solar direta no solo, alterando o microclima do local (Baretta et al., 2011). Dentre os componentes da fauna do solo, encontram-se as espécies epigéicas que vivem e se alimentam na superfície do solo, componentes da meso e macrofauna, sendo responsáveis pela fragmentação da matéria orgânica e a liberação de nutrientes, mas não pela redistribuição ativa desse material (Bignell et al., 2010).

As modificações ocorridas no solo resultantes do seu preparo podem ser avaliadas utilizando-se organismos bioindicadores da fauna epigéica. Entre os grupos de invertebrados bioindicadores que habitam o solo, destacam-se os insetos da ordem Coleoptera, principalmente, os das famílias Carabidae, Staphylinidae e Cicindelidae (Lewinsohn et al., 2005).

Portanto, o presente trabalho teve como objetivos, avaliar os efeitos de diferentes preparos do solo e coveamentos na implantação de um pomar de nogueirapecã e seu desenvolvimento inicial, no município de Santa Maria, RS, através de bioindicadores do solo, utilizando espécies da Ordem Coleoptera.

\section{Material e métodos}

O experimento foi conduzido em condições de campo, na Unidade de Pesquisa Florestal pertencente à Fundação Estadual de Pesquisa Agropecuária (2940'31's e $\left.53^{\circ} 54^{\prime} 45^{\prime} \mathrm{W}\right)$, localizada no município de Santa Maria, RS. O município está situado na região fisiográfica da Depressão Central do estado, a uma altitude média de 113 m. O clima da região, segundo Köppen, é do tipo Cfa - subtropical úmido, com verão quente (Alvares et al., 2013).

O solo da região é classificado como Argissolo Vermelho Distrófico arênico, caracterizado por apresentar-se mediamente profundo, não hidromórfico, bem drenado, apresentando horizonte Bt (do tipo B textural) avermelhado, com textura superficial franco arenosa, ácida e pobre em matéria orgânica (Streck et al., 2008).

Devido à presença das gramíneas e arbustos na área, antes da instalação dos tratamentos foi realizada em toda a área a aplicação do herbicida glifosato, bem como o controle de formigas cortadeiras com uso de iscas granuladas (sulfluramida a 3,0 g i.a. $\mathrm{kg}^{-1}$ ), aplicadas, sistematicamente, próximas às plantas e em áreas adjacentes.

As mudas utilizadas para o transplante foram produzidas a partir de sementes e, posteriormente, enxertadas com as variedades Barton e Shawnee. As mudas foram transplantadas em fevereiro de 2012 com altura padrão de $60 \mathrm{~cm}$ e idade de 2,5 anos. A adubação foi realizada em duas etapas: a primeira dose de 200 g planta $^{-1}$ de NPK com formulação 5-20-20 e $300 \mathrm{~g}$ planta $^{-1}$ de calcário dolomítico, no transplante das mudas de nogueira, incorporados ao solo na cova. Trinta dias após o plantio, as mudas receberam outra parcela de adubação de NPK utilizando-se a mesma fórmula, com base na recomendação do Manual de Adubação e Calagem (Sociedade Brasileira de Ciência do Solo, 2004). Sessenta dias após o plantio, as mudas receberam, a cada quinze dias, $20 \mathrm{~g}$ de ureia $(42 \%$ de $\mathrm{N})$, durante 60 dias.

Os tratamentos foram distribuídos em delineamento de blocos ao acaso, sendo cinco blocos, constituídos de quatro tratamentos: 1) plantio em cova pequena circular, com $20 \mathrm{~cm}$ de diâmetro e $60 \mathrm{~cm}$ de profundidade $(\mathrm{Cp})$; 2) plantio utilizando subsolador mais grade niveladora e cova pequena circular, sendo a subsolagem realizada a 40 $\mathrm{cm}$ de profundidade (Scp); 3) plantio utilizando enxada 
rotativa a uma profundidade de aproximadamente $20 \mathrm{~cm}$, e cova pequena (Ecp); 4) plantio em cova grande circular, com $40 \mathrm{~cm}$ de diâmetro e $60 \mathrm{~cm}$ de profundidade $(\mathrm{Cg})$.

Para abertura das covas pequenas, circulares de $20 \mathrm{~cm}$ de diâmetro e $60 \mathrm{~cm}$ de profundidade, foi utilizada uma broca perfuradora adaptada na motosserra $(\mathrm{Cp})$. Para abertura das covas grandes, foi utilizado um perfurador caracol mecanizado, acoplado a um trator $(\mathrm{Cg})$.

Os tratamentos foram sorteados e distribuídos nos blocos, em parcelas de $28 \mathrm{~m} \times 21 \mathrm{~m}\left(588 \mathrm{~m}^{2}\right)$, totalizando $11.760 \mathrm{~m}^{2}$ de área total. O espaçamento utilizado foi de $7 \mathrm{~m} \mathrm{x} 7 \mathrm{~m}$, com cada parcela composta de 12 plantas de nogueira-pecã, totalizando 240 plantas.

Para amostrar a fauna de Coleoptera optou-se pela utilização de armadilhas de queda tipo pitfall. As armadilhas constituíram-se de um recipiente cilíndrico de $10 \mathrm{~cm}$ de altura, com área de captura de $176,7 \mathrm{~cm}^{2}$, que continham como conservante água, cloreto de sódio e detergente $(200 \mathrm{~mL}$ de água $+15 \mathrm{~g}$ de cloreto de sódio $+2 \mathrm{~mL}$ de detergente), e eram enterradas no solo até a borda (Aquino et al., 2006). Quatro armadilhas foram instaladas na área de cada parcela dos quatro tratamentos.

Para fins de comparação, também foram realizadas coletas nas áreas do entorno, correspondentes aos quatro sentidos dos pontos cardeais: Nordeste (NE): área com aproximadamente $120 \mathrm{~m}$, em linha reta, do limite nordeste da área cercada do plantio, com presença de vegetação secundária (capoeira) e predominância de Baccharis sp. (Asteraceae) e gramíneas (Poaceae). Após a área de capoeira, encontrava-se uma área com Pinus sp., com altura média aproximada de 12 m, e Eucalytpus sp., com altura média aproximada de $15 \mathrm{~m}$; Sudeste (SU): área com aproximadamente $72 \mathrm{~m}$ em linha reta do limite sudeste do cercado do plantio, com presença de vegetação secundária (capoeira) e com predominância de Baccharis sp. (Asteraceae) e gramíneas (Poaceae). Após a capoeira, encontrava-se uma área com Eucalyptus sp., com altura média aproximada de $15 \mathrm{~m}$; Sudoeste (SO): área com aproximadamente $72 \mathrm{~m}$ em linha reta do limite sudoeste do cercado do plantio, com presença de vegetação secundária (capoeira) com predominância de Baccharis sp. (Asteraceae) e gramíneas (Poaceae). Após a capoeira, encontrava-se uma área com Eucalyptus sp., com altura média aproximada de $15 \mathrm{~m}$; Noroeste (NO): presença de área com aproximadamente $50 \mathrm{~m}$ em linha reta do limite noroeste do cercado do plantio, com presença de vegetação secundária (capoeira), com predominância das seguintes espécies: Baccharis sp. (Asteraceae) e gramíneas (Poaceae), e a presença de espécies florestais nativas como Peltophorum dubium (Spreng.) Taub. (Fabaceae) e Zanthoxylum petiolare A.St.-Hil. \& Tul. (Rutaceae).

As armadilhas foram distribuídas a $10 \mathrm{~m}$ de distância umas das outras, nas entrelinhas do primeiro quadrante de cada tratamento, em cada bloco, totalizando 80 amostras por data de coleta. As coletas foram realizadas de março de 2014 a novembro de 2015, no período correspondente à metade de cada estação climática do ano: verão (14/03/14 e 06/02/15); outono (14/05/14 e $06 / 05 / 15)$, inverno $(06 / 08 / 14$ e $10 / 08 / 15)$ e primavera $(05 / 11 / 14$ e $16 / 11 / 15)$, totalizando oito coletas e 640 amostras. Na área do entorno, nas mesmas datas de coleta dos tratamentos, foram dispostas cinco armadilhas de solo, a cerca de $30 \mathrm{~m}$ do plantio, em cada sentido dos pontos cardeais, distantes $10 \mathrm{~m}$ em linha reta umas das outras, totalizando 20 amostras por data de coleta e 160 amostras, nas oito coletas realizadas.

Após 48 h de exposição, o material coletado nas armadilhas foi recolhido em recipientes plásticos com capacidade de $80 \mathrm{~mL}$, tampados e devidamente etiquetados, e transportados até o laboratório para triagem. Em laboratório, o material coletado foi submetido à triagem com a utilização de lupas estereoscópicas. Os espécimes de Coleoptera foram morfotipados, com o auxílio de chaves dicotômicas. A seguir, os exemplares "voucher" foram transfixados e depositados na coleção do Laboratório de Entomologia Florestal da Universidade Federal de Santa Maria. As morfoespécies de Coleoptera foram encaminhadas para taxonomistas para identificação.

Foi realizada a análise dos parâmetros ecológicos para às espécies de Coleoptera. A frequência relativa (\%) foi calculada a partir do somatório dos dados das coletas, e calculada a porcentagem de indivíduos de cada espécie, em relação ao total de indivíduos coletados. A partir dos dados de frequência, determinou-se o intervalo de confiança (IC) da média com $5 \%$ de probabilidade, conforme Fazolin (1991): muito frequente (mf): número de indivíduos maior que o limite superior do IC a 5\%; frequente (f): número de indivíduos situados dentro do IC a $5 \%$; e pouco frequente (pf): número de indivíduos menor que o limite inferior do IC a 5\%.

A ddiversidade da fauna de Coleoptera nas áreas dos tratamentos no plantio de nogueira-pecã e áreas de entorno foi calculada pelo índice de diversidade de 
Shannon $\left(H^{\prime}\right)$ e o grau de uniformidade, pelo índice de equitabilidade de Pielou $\left(J^{\prime}\right)$. A fim de relacionar a diversidade da fauna de Coleoptera com as possíveis modificações ocasionadas pelos diferentes tipos de preparos do solo e coveamentos, foram realizadas as análises físicas e químicas do solo. Para a análise física, em março de 2014, realizou-se a coleta de amostras com estrutura preservada na camada de $0,0-0,10 \mathrm{~m}$ de profundidade, utilizando anéis volumétricos medindo $5,7 \mathrm{~cm}$ de diâmetro por $4 \mathrm{~cm}$ de altura, em trincheiras localizadas entre plantas de nogueira-pecã, em cada um dos tratamentos. Trabalhou-se com as propriedades físicas de densidade, macro e microporosidade e porosidade total do solo.

Para a análise química do solo, com determinação de macro e micronutrientes, foi realizada uma amostragem na área de cada tratamento, totalizando 20 amostras, bem como nas áreas adjacentes ao plantio, em cada um dos sentidos dos pontos cardeais. A coleta de solo foi realizada conforme Sociedade Brasileira de Ciência do Solo (2004), no mês de fevereiro de 2015, coincidindo com a segunda coleta da fauna de Coleoptera, referente à metade da estação climática correspondente ao verão.

Para relacionar a influência dos diferentes preparos do solo e coveamentos sobre o desenvolvimento das plantas de nogueira-pecã, foram realizadas as seguintes medições: diâmetro do coleto (Ø), a partir da circunferência, mensurada a $5 \mathrm{~cm}$ do solo, com auxílio de uma trena (mm); altura das plantas (h), do solo até a última brotação, com auxílio de uma régua telescópica (m).

As medições foram realizadas aos dois anos de idade do plantio, em março de 2014, e aos três anos de idade, em março de 2015. A diferença verificada entre as duas medições resultou no incremento médio anual (IMA).

Cabe ressaltar que todas as plantas foram mensuradas, sendo utilizadas para comparação somente as quatro plantas com desenvolvimento semelhante, ou seja, que sobreviveram desde o transplante das mudas até o momento do início das avaliações, em março de 2014. Isso porque, ao longo do experimento, foram necessários replantios, pois houve mortalidade de plantas, além de exemplares que foram retirados para a realização de outros estudos.

Com os dados obtidos nas análises químicas e físicas do solo e mensuração das plantas de nogueira-pecã, nos diferentes tipos de preparo de solo e coveamentos do plantio e sua área de entorno (apenas para as propriedades químicas e argila), foi realizada análise de variância (ANOVA), seguida do teste de Tukey, com 5\% de probabilidade de erro. Para verificar as possíveis diferenças entre a abundância da espécie mais abundante de Coleoptera, em 2014 e 2015 e no total, nos diferentes preparos de solo e coveamentos do plantio de nogueira-pecã e sua área de entorno, foi realizado o teste de Kruskal-Wallis a 5\% de probabilidade de erro. Foi também utilizado o coeficiente de correlação de Spearman $\left(r_{s}\right)$ para relacionar os valores de correlações significativas existentes entre as propriedades físicas e químicas do solo e a espécie mais abundante de Coleoptera.

\section{Resultados}

No período de março de 2014 a novembro de 2015, foi coletado um total de 552 exemplares de insetos da Ordem Coleoptera, componentes da fauna epigéica em áreas de plantio de nogueira-pecã e entorno. Deste total, 25,2\% (139 indivíduos) foram coletados na área com transplante de mudas em cova pequena $(\mathrm{Cp})$. $\mathrm{Na}$ área do entorno, considerada controle, coletou-se $24,8 \%$ (137 indivíduos), seguindo os demais tratamentos, com 19,6\% (108 indivíduos), na área com transplante de mudas em cova grande $(\mathrm{Cg}) ; 15,9 \%$ (88 indivíduos), na área com preparo do solo utilizando enxada rotativa e plantio em cova pequena (Ecp); e 14,5\% (80 indivíduos), na área com preparo do solo utilizando-se subsolador mais grade niveladora e cova pequena (Scp) (Tabela 1).

Foram observadas, no total, 60 morfoespécies de Coleoptera, sendo 32 exclusivas das áreas plantadas com nogueira-pecã, seis exclusivas da área do entorno e 22 ocorrendo nos dois ambientes. As maiores riquezas foram encontradas nas áreas do tratamento com plantio em cova grande ( $\mathrm{Cg}$ ) e entorno (En), com $S=28$ cada, e no tratamento com plantio em cova pequena $(\mathrm{Cp})$, com $S=27$. Em contrapartida, na área do tratamento Ecp foi encontrado o menor número de espécies $(S=18)$. Das 19 famílias de Coleoptera encontradas, Carabidae apresentou maior riqueza de espécies $(S=11)$ seguida das famílias Chrysomelidae, Curculionidae, Scarabaeidae e Staphylinidae, todas $\operatorname{com} S=7$; Histeridade, $\operatorname{com} S=3$; Anthicidae, Brachyderidae, Elateridae Ptilodactylidae e Tenebrionidade, todas $\operatorname{com} S=2$; e, por fim, Buprestidae, Dryophthoridae, Geotrupidae, Limnichidae, Lycidae, Meloidae, Melolonthidae e Nitidulidae, $\operatorname{com} S=1$ (Tabela 1). 
Tabela 1. Abundância de insetos adultos da Ordem Coleoptera coletados com armadilhas de solo tipo pitfall, em áreas de plantio de nogueira-pecã submetido a diferentes preparos do solo e coveamentos, e área do entorno, no período de março de 2014 a novembro de 2015, em Santa Maria, RS.

\begin{tabular}{|c|c|c|c|c|c|c|}
\hline \multirow{2}{*}{ Famílias/Espécies } & \multicolumn{4}{|c|}{ Tratamentos $^{1}$} & \multirow{2}{*}{$\mathbf{E n}^{2}$} & \multirow{2}{*}{$\begin{array}{l}\text { Abundância } \\
\text { N (\%) }\end{array}$} \\
\hline & $\mathrm{Cp}$ & Scp & Ecp & $\mathrm{Cg}$ & & \\
\hline \multicolumn{7}{|l|}{ Anthicidae } \\
\hline Acanthinus sp.1 & - & - & - & 1 & 1 & $2(0,4) \mathrm{pf}$ \\
\hline Acanthinus sp.2 & 1 & - & - & 2 & - & $3(0,5) \mathrm{pf}$ \\
\hline \multicolumn{7}{|l|}{ Brachyderidae } \\
\hline Erirrhininae sp.1 & 2 & - & - & 3 & - & $5(0,9) \mathrm{pf}$ \\
\hline Erirrhininae sp.2 & - & 5 & 2 & - & - & $7(1,3) \mathrm{pf}$ \\
\hline \multicolumn{7}{|l|}{ Buprestidae } \\
\hline Taphrocerus sp.1 & - & 1 & - & - & - & $1(0,2) \mathrm{pf}$ \\
\hline \multicolumn{7}{|l|}{ Carabidae } \\
\hline Ardistomis sp.1 & 2 & - & - & 1 & - & $3(0,5) \mathrm{pf}$ \\
\hline Blennidus sp.1 & 4 & 2 & 6 & 5 & 14 & $31(5,6) \mathrm{pf}$ \\
\hline Distichus (Distichus) sp.1 & 12 & 2 & 8 & 5 & 5 & $32(5,8) \mathrm{pf}$ \\
\hline Marsyas sp.1 & - & 1 & - & - & - & $1(0,2) \mathrm{pf}$ \\
\hline $\begin{array}{l}\text { Megacephala (Tetracha) } \\
\text { brasiliensis (Kirby, 1818) }\end{array}$ & 7 & 3 & & 3 & 5 & $20(3,6) \mathrm{pf}$ \\
\hline Notiobia (Anisotarsus) sp.1 & 6 & 2 & 4 & 4 & 2 & $18(3,3) \mathrm{pf}$ \\
\hline Notiobia (Anisotarsus) sp.2 & - & - & - & 1 & - & $1(0,2) \mathrm{pf}$ \\
\hline $\begin{array}{l}\text { Polpochila (Polpochila) } \\
\text { impressifrons Dejean, } 1831\end{array}$ & - & - & 1 & - & - & $1(0,2) \mathrm{pf}$ \\
\hline Stenolophus sp. 1 & 4 & 2 & - & 1 & 25 & $32(5,8) \mathrm{pf}$ \\
\hline Stenolophus sp.2 & 6 & 1 & 3 & 5 & 5 & $20(3,6) \mathrm{pf}$ \\
\hline Stenolophus sp. 3 & 3 & - & 2 & - & - & $5(0,9) \mathrm{pf}$ \\
\hline \multicolumn{7}{|l|}{ Chrysomelidae } \\
\hline $\begin{array}{l}\text { Bradycassis drewseni } \\
\text { (Boheman, 1855) }\end{array}$ & - & 2 & 1 & 1 & 2 & $6(1,1) \mathrm{pf}$ \\
\hline Caraguata sp.1 & - & - & 1 & 2 & - & $3(0,5) \mathrm{pf}$ \\
\hline Chaetocnema sp.1 & - & 1 & - & - & - & $1(0,2) \mathrm{pf}$ \\
\hline Colaspis sp.1 & - & 2 & - & - & - & $2(0,4) \mathrm{pf}$ \\
\hline Systena sp.1 & 2 & - & - & - & - & $2(0,4) \mathrm{pf}$ \\
\hline Chrysomelini sp. 1 & 3 & 3 & - & 2 & 1 & $9(1,6) \mathrm{pf}$ \\
\hline Eumolpini sp.1 & - & - & - & - & 1 & $1(0,2) \mathrm{pf}$ \\
\hline \multicolumn{7}{|l|}{ Curculionidae } \\
\hline Asynonychus sp.1 & 1 & - & - & - & - & $1(0,2) \mathrm{pf}$ \\
\hline Byzes sp.1 & - & - & - & - & 1 & $1(0,2) \mathrm{pf}$ \\
\hline Conotrachelus sp.1 & - & - & - & 1 & - & $1(0,2) \mathrm{pf}$ \\
\hline Pantomorus sp.1 & - & - & - & - & 1 & $1(0,2) \mathrm{pf}$ \\
\hline Pantomorus sp.2 & 2 & 2 & - & - & 3 & $7(1,3) \mathrm{pf}$ \\
\hline Apostasimerini sp.1 & - & 1 & - & - & 5 & $6(1,1) \mathrm{pf}$ \\
\hline Apostasimerini sp. 2 & - & - & - & - & 2 & $2(0,4) \mathrm{pf}$ \\
\hline \multicolumn{7}{|l|}{ Dryophthoridae } \\
\hline $\begin{array}{l}\text { Sphenophorus brunnipennis } \\
\text { (Germar, 1824) }\end{array}$ & - & 1 & - & 2 & 1 & $4(0,7) \mathrm{pf}$ \\
\hline \multicolumn{7}{|l|}{ Elateridae } \\
\hline $\begin{array}{l}\text { Conoderus alfredoi G. Tomé, } \\
1995\end{array}$ & - & - & - & 3 & - & $3(0,5) \mathrm{pf}$ \\
\hline Heteroderes sp.1 & - & - & - & 2 & 1 & $3(0,5) \mathrm{pf}$ \\
\hline \multicolumn{7}{|l|}{ Geotrupidae } \\
\hline $\begin{array}{l}\text { Neoathyreus lanuginosus } \\
\text { (Klug, 1843) }\end{array}$ & 2 & - & - & - & - & $2(0,4) \mathrm{pf}$ \\
\hline
\end{tabular}

Continua...
Tabela 1. continuação.

\begin{tabular}{|c|c|c|c|c|c|c|}
\hline \multirow{2}{*}{ Famílias/Espécies } & \multicolumn{4}{|c|}{ Tratamentos $^{1}$} & \multirow{2}{*}{$\mathbf{E n}^{2}$} & \multirow{2}{*}{$\begin{array}{c}\text { Abundância } \\
\text { N (\%) }{ }^{3}\end{array}$} \\
\hline & $\mathrm{Cp}$ & Scp & Ecp & $\mathrm{Cg}$ & & \\
\hline \multicolumn{7}{|l|}{ Histeridae } \\
\hline Euspilotus (Euspilotus) sp.1 & - & - & - & 2 & - & $2(0,4) \mathrm{pf}$ \\
\hline Operclipygus sp.1 & 1 & - & - & - & - & $1(0,2) \mathrm{pf}$ \\
\hline Exosternini sp.1 & - & 1 & - & - & - & $1(0,2) \mathrm{pf}$ \\
\hline \multicolumn{7}{|l|}{ Limnichidae } \\
\hline Corrinea sp. 1 & - & - & 2 & - & - & $2(0,4) \mathrm{pf}$ \\
\hline \multicolumn{7}{|l|}{ Lycidae } \\
\hline Idiopteron sp. 1 & - & 1 & 2 & - & - & $3(0,5) \mathrm{pf}$ \\
\hline \multicolumn{7}{|l|}{ Meloidae } \\
\hline Epicauta sp.1 & 1 & 1 & - & - & - & $2(0,4) \mathrm{pf}$ \\
\hline \multicolumn{7}{|l|}{ Melolonthidae } \\
\hline $\begin{array}{l}\text { Diloboderus abderus (Sturm, } \\
1826 \text { ) }\end{array}$ & 55 & 41 & 47 & 43 & 40 & $226(40,9) \mathrm{mf}$ \\
\hline \multicolumn{7}{|l|}{ Nitidulidae } \\
\hline Lasiodactylus sp. 1 & 8 & 2 & - & 2 & 1 & $13(2,4) \mathrm{pf}$ \\
\hline \multicolumn{7}{|l|}{ Ptilodactylidae } \\
\hline Ptilodactyla sp.1 & 4 & - & - & 2 & - & $6(1,1) \mathrm{pf}$ \\
\hline Ptilodactyla sp. 2 & - & - & 1 & - & 2 & $3(0,5) \mathrm{pf}$ \\
\hline \multicolumn{7}{|l|}{ Scarabaeidae } \\
\hline $\begin{array}{l}\text { Ataenius opatrinus Harold, } \\
1867\end{array}$ & - & - & - & - & 2 & $2(0,4) \mathrm{pf}$ \\
\hline $\begin{array}{l}\text { Ataenius platensis (Blanchard, } \\
1846 \text { ) }\end{array}$ & 2 & - & 3 & 1 & 5 & $11(2,0) \mathrm{pf}$ \\
\hline $\begin{array}{l}\text { Canthon (Canthon) lituratus } \\
\text { (Germar, 1813) }\end{array}$ & 1 & - & - & - & 2 & $3(0,5) \mathrm{pf}$ \\
\hline $\begin{array}{l}\text { Canthon (Canthon) virens } \\
\text { chalybaeus Blanchard, } 1845\end{array}$ & 2 & - & 1 & 1 & 3 & $7(1,3) \mathrm{pf}$ \\
\hline Canthon (Canthon) sp.1 & 1 & - & - & - & - & $1(0,2) \mathrm{pf}$ \\
\hline $\begin{array}{l}\text { Deltochilum (Deltohyboma) } \\
\text { sculpturatum Felsche, } 1907\end{array}$ & - & 1 & 1 & - & - & $2(0,4) \mathrm{pf}$ \\
\hline Uroxys sp. 1 & - & - & - & - & 3 & $3(0,5) \mathrm{pf}$ \\
\hline \multicolumn{7}{|l|}{ Staphylinidae } \\
\hline Atheta sp.1 & - & 1 & - & - & - & $1(0,2) \mathrm{pf}$ \\
\hline Atheta sp.2 & - & 1 & - & - & - & $1(0,2) \mathrm{pf}$ \\
\hline $\begin{array}{l}\text { Eulissus chalybaeus } \\
\text { Mannerheim, } 1830\end{array}$ & - & - & - & 2 & - & $2(0,4) \mathrm{pf}$ \\
\hline Hamotus sp.1 & - & - & - & 1 & - & $1(0,2) \mathrm{pf}$ \\
\hline Osorius sp.1 & 2 & - & - & 8 & 2 & $12(2,2) \mathrm{pf}$ \\
\hline Quedius sp.1 & - & - & - & 2 & - & $2(0,4) \mathrm{pf}$ \\
\hline Aleocharinae sp.1 & 4 & - & - & - & 1 & $5(0,9) \mathrm{pf}$ \\
\hline \multicolumn{7}{|l|}{ Tenebrionidae } \\
\hline $\begin{array}{l}\text { Lagria villosa (Fabricius, } \\
\text { 1783) }\end{array}$ & 1 & - & - & - & - & $1(0,2) \mathrm{pf}$ \\
\hline Strongylium $\mathrm{sp} .1$ & - & - & 1 & - & 1 & $2(0,4) \mathrm{pf}$ \\
\hline Abundância (N) & 139 & 80 & 88 & 108 & 137 & 552 \\
\hline Riqueza $(S)$ & 27 & 24 & 18 & 28 & 28 & 60 \\
\hline Diversidade de Shannon $\left(H^{\prime}\right)$ & 2,48 & 2,16 & 1,89 & 2,54 & 2,55 & 2,72 \\
\hline Equitabilidade de Pielou $\left(J^{\prime}\right)$ & 0,75 & 0,68 & 0,66 & 0,76 & 0,77 & 0,66 \\
\hline \multicolumn{7}{|c|}{$\begin{array}{l}{ }^{1} \text { Tratamentos: } \mathrm{Cp}=\text { plantio em cova pequena }(20 \mathrm{~cm} \times 60 \mathrm{~cm}) ; \mathrm{Scp}=\text { utilização } \\
\text { de subsolador mais grade niveladora e cova pequena; Ecp = utilização de } \\
\text { enxada rotativa e cova pequena; e, } \mathrm{Cg}=\text { plantio em cova grande }(40 \mathrm{~cm} \times \\
60 \mathrm{~cm}) ;{ }^{2} \mathrm{En}=\text { somatório de indivíduos coletados nas áreas adjacentes ao } \\
\text { plantio de nogueira-pecã (nordeste, sudeste, sudoeste e noroeste); }{ }^{3} \text { Número } \\
\text { total de indivíduos = N; Frequência relativa }(\%): \mathrm{mf}=\text { muito frequente, } \mathrm{f}= \\
\text { frequente e pf = pouco frequente. }\end{array}$} \\
\hline
\end{tabular}

Pesq. flor. bras., Colombo, v. 37, n. 92, p. 587-596, out./dez. 2017 
Da riqueza total de espécies de Coleoptera encontradas, que apresentaram as maiores frequências (maior que 5,0\%), foram Diloboderus abderus (Sturm) (Melolonthidae), seguida das espécies Distichus (Distichus) sp.1, Stenolophus sp.1 e Blennidus sp.1 (Carabidae). Os indivíduos adultos de D. abderus contabilizados neste estudo foram observados apenas nas coletas realizadas no verão, em 14 de março de 2014 e 06 de fevereiro de 2015 (Tabela 1). Não foi verificada diferença entre as médias de abundância entre os tratamentos e área do entorno (Kruskal-Wallis, $\mathrm{p} \geq 0,05, \mathrm{n}=100$ ) da espécie de D. abderus (Figura 1).

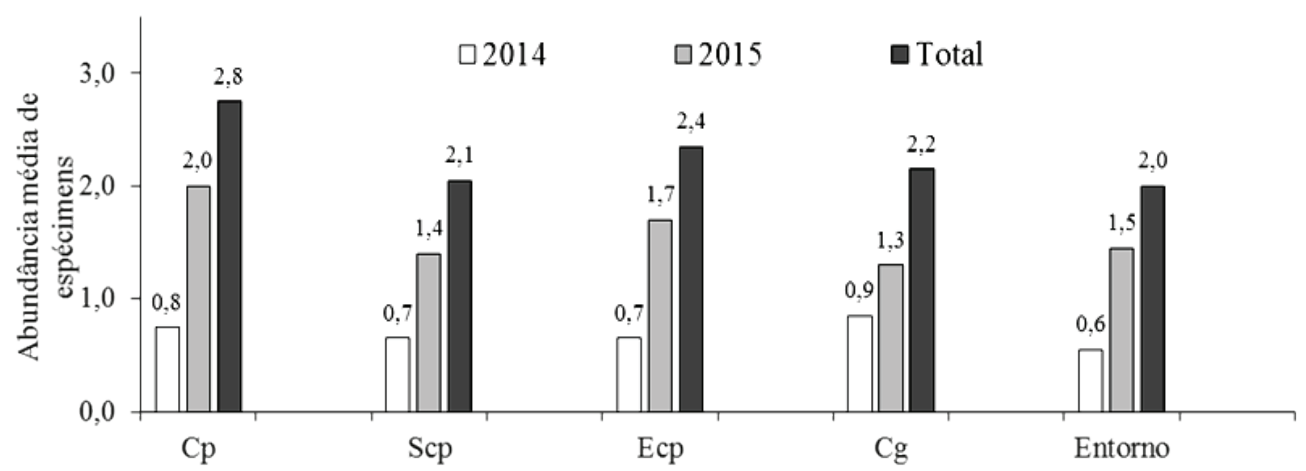

Figura 1. Abundância média de adultos de Diloboderus abderus (Sturm), coletados nos diferentes preparos de solo e coveamentos: em cova pequena de $20 \mathrm{~cm}$ x $60 \mathrm{~cm}(\mathrm{Cp})$; subsolador mais grade niveladora e cova pequena (Scp); enxada rotativa e cova pequena (Ecp); em cova grande de 40 cm x 60 cm (Cg): em um plantio de nogueira-pecã e área do entorno, em 14 de março de 2014 e 06 de fevereiro de 2015, em Santa Maria, RS.

A partir dos dados de análise das propriedades físicas do solo, não se verificou diferença significativa (ANOVA, $\mathrm{p} \geq 0,05$ ), para os diferentes tratamentos avaliados, bem como para a área do entorno. Os valores médios encontrados para a porcentagem de argila variaram de $16,8 \%$ no tratamento Scp e $17,8 \%$, no tratamento Cg e área do entorno (En) (Tabela 2).

Apesar dos tratamentos não diferirem estatisticamente entre si para as propriedades físicas do solo (Tabela 2), possivelmente pelo tempo transcorrido desde o preparo do solo, observou-se correlação significativa positiva para a abundância de Diloboderus abderus e a porcentagem de argila encontrada no solo $\left(r_{s}=0,763\right.$, $\mathrm{p} \leq 0,01)$.

Para as propriedades químicas, assim como para a matéria orgânica não foram observadas diferenças significativas (ANOVA, $\mathrm{p} \geq 0,05$ ) entre as médias encontradas nos diferentes preparos de solo e coveamentos (Tabela 3 ).

Cabe ressaltar, ainda, os baixos valores de $\mathrm{pH}$ e $\mathrm{Zn}$, encontrados no solo em todas as áreas dos tratamentos (Tabela 3). D. abderus apresentou correlação significativa positiva entre a abundância e o $\mathrm{pH}$ do solo $\left(r_{s}=0,627, \mathrm{p}<0,01\right)$ e correlação significativa negativa entre a abundância e $\mathrm{Al}\left(r_{s}=-0,428, \mathrm{p}<0,05\right)$.
Os valores médios da mensuração do crescimento das plantas de nogueira-pecã, no segundo (2014) e no terceiro (2015) ano após o plantio, referentes ao diâmetro à altura do coleto e a altura das plantas, e o incremento para esses dois parâmetros encontram-se descritos na Tabela 4.

Pode-se observar na Tabela 4 que houve diferença significativa (ANOVA, $\mathrm{p} \leq 0,05$ ) para o diâmetro e para a altura entre os anos de 2014 e 2015, sendo os maiores valores verificados para 2015, em todos os tratamentos. O tratamento com plantio em cova pequena $(\mathrm{Cp})$ apresentou menor diâmetro médio à altura do coleto em 2015 ( $\varnothing=34,1 \mathrm{~mm})$, enquanto que o maior diâmetro $(\varnothing=44,7 \mathrm{~mm})$, no mesmo ano, foi encontrado para o tratamento com plantio em cova grande.

$\mathrm{O}$ incremento médio anual (IMA) refere-se à diferença encontrada entre 2014 e 2015, para valores de diâmetro à altura do coleto e altura das plantas de nogueira-pecã. Os valores de IMA para altura das plantas apresentou diferença estatística (ANOVA, $\mathrm{p} \leq 0,05$ ), sendo a maior média verificada para o tratamento $\mathrm{Cp}$ e a menor para o tratamento Cg. Ainda assim, as plantas de nogueira-pecã, nesse último tratamento, apresentaram maior altura e diâmetro em detrimento de Cp (Tabela 4). 
Tabela 2. Porcentagem de argila, densidade, macro e microporosidade e porosidade total do solo, na profundidade de $0,0-0,10 \mathrm{~m}$, em diferentes preparos de solo e coveamentos em um plantio de nogueira-pecã e área do entorno em fevereiro de 2015, em Santa Maria, RS.

\begin{tabular}{cccccc}
\hline Tratamentos $^{1}$ & $\begin{array}{c}\text { Argila } \\
(\%)\end{array}$ & $\begin{array}{c}\text { Densidade do } \\
\text { Solo } \\
\left(\mathbf{g ~ c m}^{-3}\right)\end{array}$ & \multicolumn{3}{c}{ Morosidade $\left(\mathbf{c m}^{3} \mathbf{c m}^{-3}\right)$} \\
Mp & $17,3 \mathrm{~A}^{3}$ & $1,57 \mathrm{~A}$ & $0,16 \mathrm{~A}$ & $0,25 \mathrm{~A}$ & $0,40 \mathrm{~A}$ \\
$\mathrm{Scp}$ & $16,8 \mathrm{~A}$ & $1,53 \mathrm{~A}$ & $0,15 \mathrm{~A}$ & $0,27 \mathrm{~A}$ & $0,42 \mathrm{~A}$ \\
$\mathrm{Ecp}$ & $17,6 \mathrm{~A}$ & $1,46 \mathrm{~A}$ & $0,21 \mathrm{~A}$ & $0,25 \mathrm{~A}$ & $0,46 \mathrm{~A}$ \\
$\mathrm{Cg}$ & $17,8 \mathrm{~A}$ & $1,61 \mathrm{~A}$ & $0,14 \mathrm{~A}$ & $0,28 \mathrm{~A}$ & $0,42 \mathrm{~A}$ \\
$\mathrm{En}^{2}$ & $17,8 \mathrm{~A}$ & - & - & - & - \\
\hline $\mathrm{CV}(\%)$ & 7,9 & 6,2 & 11,6 & 9,1 & 7,7 \\
\hline
\end{tabular}

${ }^{1}$ Tratamentos: $\mathrm{Cp}=$ plantio em cova pequena $(20 \mathrm{~cm}$ x $60 \mathrm{~cm}) ; \mathrm{Scp}=$ utilização de subsolador mais grade niveladora e cova pequena; Ecp = utilização de enxada rotativa e cova pequena; e $\mathrm{Cg}=$ plantio em cova grande $(40 \mathrm{~cm} \times$ $60 \mathrm{~cm}) ;{ }^{2} \mathrm{En}=$ somatório de indivíduos coletados nas áreas adjacentes ao plantio de nogueira-pecã (nordeste, sudeste, sudoeste e noroeste). ${ }^{3}$ Médias seguidas da mesma letra maiúscula, na coluna, não diferem entre si pelo teste de Tukey a $5 \%$ de significância. $(n=24$ para argila e $n=20$ para as demais propriedades físicas).

Tabela 3. Matéria orgânica (MO), potencial hidrogeniônico $(\mathrm{pH})$, micronutrientes ( $\mathrm{B}, \mathrm{Cu}$ e $\mathrm{Zn})$ e macronutrientes ( $\mathrm{P}, \mathrm{S}, \mathrm{K}$, $\mathrm{Al}, \mathrm{Ca}$ e $\mathrm{Mg}$ ), em diferentes preparos de solo e coveamentos em um plantio de nogueira-pecã e área do entorno em fevereiro de 2015, em Santa Maria, RS.

\begin{tabular}{|c|c|c|c|c|c|c|}
\hline \multirow{3}{*}{ Tratamentos ${ }^{1}$} & \multirow{3}{*}{$\begin{array}{l}\text { MO } \\
\text { (\%) }\end{array}$} & \multirow{3}{*}{\multicolumn{2}{|c|}{$\begin{array}{c}\text { pH } \\
\left(\mathbf{H}_{2} \mathrm{O}\right)\end{array}$}} & \multicolumn{3}{|c|}{ Micronutrientes } \\
\hline & & & & B & $\mathrm{Cu}$ & Zn \\
\hline & & & & \multicolumn{3}{|c|}{$\left(\mathrm{mg} \mathrm{dm}^{-3}\right)$} \\
\hline $\mathrm{Cp}$ & \multicolumn{2}{|c|}{$2,3 \mathrm{~A}^{3}$} & $5,1 \mathrm{~A}$ & $0,5 \mathrm{~A}$ & $1,3 \mathrm{~A}$ & $1,1 \mathrm{~A}$ \\
\hline Scp & \multicolumn{2}{|l|}{$1,9 \mathrm{~A}$} & $5,0 \mathrm{~A}$ & $0,6 \mathrm{~A}$ & $1,4 \mathrm{~A}$ & $1,0 \mathrm{~A}$ \\
\hline Ecp & \multicolumn{2}{|l|}{$1,5 \mathrm{~A}$} & $5,0 \mathrm{~A}$ & $0,5 \mathrm{~A}$ & $1,2 \mathrm{~A}$ & $0,5 \mathrm{~A}$ \\
\hline $\mathrm{Cg}$ & \multicolumn{2}{|c|}{$2,2 \mathrm{~A}$} & $5,0 \mathrm{~A}$ & $0,6 \mathrm{~A}$ & $1,5 \mathrm{~A}$ & $1,3 \mathrm{~A}$ \\
\hline $\mathrm{En}^{2}$ & \multicolumn{2}{|l|}{$1,7 \mathrm{~A}$} & $4,9 \mathrm{~A}$ & $0,5 \mathrm{~A}$ & $1,3 \mathrm{~A}$ & $1,1 \mathrm{~A}$ \\
\hline \multirow[t]{2}{*}{ CV (\%) } & \multicolumn{2}{|l|}{11,3} & 3,1 & 17,1 & 17,6 & 27,4 \\
\hline & \multicolumn{6}{|c|}{ Macronutrientes } \\
\hline \multirow[t]{2}{*}{ Tratamentos $^{1}$} & $\mathbf{P}$ & $\mathbf{S}$ & $\mathbf{K}$ & Al & $\mathrm{Ca}$ & Mg \\
\hline & \multicolumn{3}{|c|}{$\left(\mathrm{mg} \mathrm{dm}^{-3}\right)$} & \multicolumn{3}{|c|}{$\left(\mathrm{cmol}_{\mathrm{c}} \mathrm{dm}^{-3}\right)$} \\
\hline $\mathrm{Cp}$ & $6,7 \mathrm{~A}^{3}$ & $14,2 \mathrm{~A}$ & $103,5 \mathrm{~A}$ & $0,3 \mathrm{~A}$ & $2,6 \mathrm{~A}$ & $1,0 \mathrm{~A}$ \\
\hline Scp & $6,1 \mathrm{~A}$ & $14,7 \mathrm{~A}$ & $91,3 \mathrm{~A}$ & $0,3 \mathrm{~A}$ & $2,2 \mathrm{~A}$ & $0,9 \mathrm{~A}$ \\
\hline Ecp & $4,8 \mathrm{~A}$ & $13,3 \mathrm{~A}$ & $87,3 \mathrm{~A}$ & $0,3 \mathrm{~A}$ & $2,1 \mathrm{~A}$ & $0,9 \mathrm{~A}$ \\
\hline $\mathrm{Cg}$ & $6,7 \mathrm{~A}$ & $13,6 \mathrm{~A}$ & $80,9 \mathrm{~A}$ & $0,4 \mathrm{~A}$ & $2,6 \mathrm{~A}$ & $0,9 \mathrm{~A}$ \\
\hline $\mathrm{En}^{2}$ & $3,9 \mathrm{~A}$ & $12,8 \mathrm{~A}$ & $63,0 \mathrm{~A}$ & $0,5 \mathrm{~A}$ & $2,0 \mathrm{~A}$ & $0,8 \mathrm{~A}$ \\
\hline CV (\%) & 16,8 & 11,5 & 15,4 & 16,8 & 8,9 & 17,6 \\
\hline \multicolumn{7}{|c|}{$\begin{array}{l}{ }^{1} \text { Tratamentos: } \mathrm{Cp}=\text { plantio em cova pequena }(20 \mathrm{~cm} \times 60 \mathrm{~cm}) ; \mathrm{Scp}=\text { utilização } \\
\text { de subsolador mais grade niveladora e cova pequena; Ecp = utilização de } \\
\text { enxada rotativa e cova pequena; e } \mathrm{Cg}=\text { plantio em cova grande }(40 \mathrm{~cm} \times 60 \\
\mathrm{cm}) ;{ }^{2} \mathrm{En}=\text { somatório de indivíduos coletados nas áreas adjacentes ao plantio } \\
\text { de nogueira-pecã (nordeste, sudeste, sudoeste e noroeste). }{ }^{3} \text { Médias seguidas da } \\
\text { mesma letra maiúscula, na coluna, não diferem entre si pelo teste de Tukey } \\
\text { a } 5 \% \text { de significância. }\end{array}$} \\
\hline
\end{tabular}

Tabela 4. Valores médios de diâmetro do coleto (Ø), altura (h) e incremento médio anual (IMA) de plantas de nogueirapecã, em um plantio submetido a diferentes preparos de solo e coveamentos, em março de 2014 e de 2015, em Santa Maria, RS.

\begin{tabular}{|c|c|c|c|c|c|c|}
\hline \multirow{3}{*}{ Trat. } & \multicolumn{2}{|c|}{ Março de 2014} & \multicolumn{2}{|c|}{ Março de 2015} & \multicolumn{2}{|c|}{ IMA } \\
\hline & $\varnothing(\mathrm{mm})$ & h (m) & $\varnothing(\mathrm{mm})$ & h (m) & $\varnothing(\mathrm{mm})$ & h (m) \\
\hline & \multicolumn{2}{|c|}{ Média $\pm \sigma$} & \multicolumn{2}{|c|}{ Média $\pm \sigma$} & \multicolumn{2}{|c|}{ Média $\pm \sigma$} \\
\hline $\mathrm{Cp}$ & $\begin{array}{l}22,8 \pm \\
2,9 \mathrm{bA}\end{array}$ & $\begin{array}{c}1,9 \pm \\
0,3 \mathrm{bA}\end{array}$ & $\begin{array}{l}34,1 \pm \\
5,5 \mathrm{aA}\end{array}$ & $\begin{array}{c}2,8 \pm 0,5 \\
\mathrm{aA}\end{array}$ & $\begin{array}{c}11,3 \pm \\
4,8 \mathrm{~A}\end{array}$ & $\begin{array}{l}1,1 \pm \\
0,3 \mathrm{~A}\end{array}$ \\
\hline Scp & $\begin{array}{l}23,4 \pm \\
4,9 \mathrm{bA}\end{array}$ & $\begin{array}{c}1,9 \pm \\
0,6 \mathrm{bA}\end{array}$ & $\begin{array}{c}40,4 \pm 6,6 \\
\mathrm{aAB}\end{array}$ & $\begin{array}{c}3,0 \pm 0,5 \\
\mathrm{aA}\end{array}$ & $\begin{array}{c}17,0 \pm \\
7,0 \mathrm{~A}\end{array}$ & $\begin{array}{c}1,1 \pm \\
0,5 \mathrm{AB}\end{array}$ \\
\hline Ecp & $\begin{array}{l}22,3 \pm \\
3,4 \mathrm{bA}\end{array}$ & $\begin{array}{c}2,0 \pm \\
0,3 \mathrm{bA}\end{array}$ & $\begin{array}{c}37,4 \pm 5,3 \\
\mathrm{aAB}\end{array}$ & $\begin{array}{c}2,7 \pm 0,4 \\
\mathrm{aA}\end{array}$ & $\begin{array}{c}15,1 \pm \\
4,6 \mathrm{~A}\end{array}$ & $\begin{array}{r}0,8 \pm \\
0,3 \mathrm{AB}\end{array}$ \\
\hline $\mathrm{Cg}$ & $\begin{array}{l}27,7 \pm \\
4,6 \mathrm{bA}\end{array}$ & $\begin{array}{c}2,3 \pm \\
0,4 \mathrm{bA}\end{array}$ & $\begin{array}{l}44,7 \pm \\
9,4 \mathrm{aB}\end{array}$ & $\begin{array}{c}3,1 \pm 0,4 \\
\mathrm{aA}\end{array}$ & $\begin{array}{c}17,1 \pm \\
6,4 \mathrm{~A}\end{array}$ & $\begin{array}{l}0,8 \pm \\
0,3 \mathrm{~B}\end{array}$ \\
\hline
\end{tabular}

Tratamentos: $\mathrm{Cp}=$ plantio em cova pequena $(20 \mathrm{~cm} \times 60 \mathrm{~cm}) ; \mathrm{Scp}=$ utilização de subsolador mais grade niveladora e cova pequena; Ecp = utilização de enxada rotativa e cova pequena; e $\mathrm{Cg}=$ plantio em cova grande $(40 \mathrm{~cm}$ x 60 $\mathrm{cm})$. Médias seguidas da mesma letra minúscula nas linhas e maiúscula na coluna, não diferem entre si pelo teste de Tukey a $5 \%$ de significância $(n=80)$.

\section{Discussão}

Apresentando estreita relação com o solo, muitas espécies de Coleoptera apresentam uma ou todas as fases do desenvolvimento sob a terra, constituindo-se assim como bons bioindicadores ambientais. Nesse aspecto, a riqueza, geralmente, está relacionada à disponibilidade de recursos e das alterações abióticas (Townsend et al., 2006). Assim, os índices de diversidade de Shannon e equitabilidade de Pielou, encontrados para Coleoptera no tratamento utilizando enxada rotativa, indicam que houve uma alteração na disponibilidade dos recursos, causando efeitos negativos em longo prazo (dois anos após a implantação do plantio), sobre a fauna de Coleopotera, em detrimento dos demais tratamentos.

Em áreas com diferentes usos do solo, a diversidade de Coleoptera presente no solo pode estar associada, entre outros fatores, com a maior diversidade de recursos alimentares (Nunes et al., 2009); com a disponibilidade de matéria orgânica (Marques et al., 2014); com os efeitos da fragmentação florestal (Cajaiba \& Silva, 2015); e com uma maior diversidade vegetal (Garlet et al., 2015).

Silva \& Silva (2011) destacam para a ordem Coleoptera, as famílias Carabidae, Coccinellidae, Staphylinidae e Scarabaeidae (stricto sensu), por constituírem-se como importantes bioindicadores da 
qualidade e degradação ambiental, de forma que podem ser utilizadas em diferentes ecossistemas, pelas funções que desempenham no ambiente, bem como por sua estreita relação com o mesmo, além de apresentarem sensibilidade às mudanças ambientais. Cajaiba \& Silva (2015), utilizando armadilha de queda modelo pitfall, encontraram maior riqueza para a família Carabidae, em detrimento das demais. $\mathrm{O}$ hábito predador da maior parte das espécies de Carabidae torna a família importante (Forti et al., 2011), uma vez que espécies predadoras podem atuar no controle biológico natural de pragas. No presente estudo, esta família apresentou maior riqueza de espécies. Dentre as espécies de maior frequência encontradas com papel ecológico significativo, destacamse: Blennidus sp. 1 pertencente à tribo Pterostichini, que engloba espécies predadoras de pequenos artrópodes; Distichus (Distichus) sp.1, pertencente à subfamília Scaritinae, sendo composta de espécies predadoras de larvas de coleópteros, lepidópteros e de outros pequenos artrópodes; Stenolophus sp.1, pertencente à tribo Harpalini, constituída de espécies herbívoras espermófagas, em que larvas e adultos alimentam-se de sementes e frutos e vivem no folhiço sob gramíneas (Marinoni et al., 2001).

No entanto, a espécie mais abundante encontrada no presente estudo, Diloboderus abderus (Sturm), é conhecida popularmente como coró-das-pastagens. É uma espécie univoltina e polífaga, reportada no Rio Grande do Sul, na Argentina e no Uruguai, causando danos às culturas agrícolas e pastagens. Os adultos desta espécie podem ser encontrados de novembro a abril, com maior frequência em janeiro e fevereiro, períodos em que ocorre o pico de oviposição (Salvadori \& Pereira, 2006). Fato corroborado pela ocorrência de D. abderus no presente estudo somente nas coletas de março de 2014 e fevereiro de 2015.

De acordo com Silva \& Salvadori (2004), as larvas de D. abderus constroem galerias no solo e, principalmente, no terceiro ínstar causam danos ao consumirem sementes, raízes e partes verdes das plantas, as quais são carregadas para as galerias. Segundo os autores, a incidência do inseto está associada ao sistema de plantio direto, devido aos restos culturais que estão ligados aos requisitos biológicos da espécie.

Dessa forma, no presente estudo o manejo utilizado pode ter favorecido a maior ocorrência da espécie nas áreas avaliadas do plantio, uma vez que roçadas mecânicas de limpeza foram realizadas constantemente, e os restos das plantas infestantes permaneciam depositadas sobre o solo. Segundo Correia \& Oliveira (2005), a prática do plantio direto promove um ambiente favorável ao desenvolvimento do coró, uma vez que assegura alimento, microclima propício e, como não há preparo do solo, as galerias construídas pelas larvas não são destruídas. Esses fatores se aplicam também à área do entorno, o que é corroborado pela abundância encontrada nessa área.

Garlet et al. (2009) registraram o ataque de larvas de $D$. abderus em mudas clonais de eucalipto, em um plantio com 45 dias. A injúria, segundo os autores, foi causada pela larva ao consumir todo o entorno do caule das plantas (na altura do coleto), resultando na morte das mudas. No presente estudo não foi verificado ataque da espécie em mudas de C. illinoinensis, mesmo essa se apresentando muito frequente nos locais de plantio. Apesar de não terem sido verificados danos às plantas, D. abderus pode constituir um risco potencial de praga para a pecanicultura.

Os resultados demonstraram que os tratamentos em área de nogueira-pecã, com diferentes preparos do solo e coveamentos, dois anos após realizado o plantio, pouco interferiram nos atributos físicos do solo. As porcentagens de argila encontradas nos tratamentos caracterizaram a textura média, que é considerada a melhor para implantação de C. illinoinensis (Baker \& Broadfoot, 1979). Segundo Streck et al. (2008), a classe de solo Argissolo Vermelho Distrófico arênico possui no horizonte A cerca de $10 \%$ de argila.

Estudos realizados em diferentes ambientes indicaram que tanto as propriedades físicas, quanto as propriedades químicas do solo podem interferir na composição dos organismos componentes da fauna edáfica (Baretta et al., 2006; Lima et al., 2010; Portilho et al., 2011). Fato corroborado pela correlação significativa positiva para a abundância de $D$. abderus e a porcentagem de argila encontrada no solo. Garlet et al. (2009) verificaram maior ocorrência de ataque de $D$. abderus em mudas de eucalipto, em áreas de solo argiloso.

No presente estudo, os valores de densidade do solo apresentaram-se dentro dos limites da média recomendada por Baker \& Broadfoot (1979), que é de $1,4-1,7 \mathrm{~g} \mathrm{~cm}^{-3}$. No entanto, os mesmos autores indicam como o melhor para o cultivo de C. illinoinensis, valores menores que $1,4 \mathrm{~g} \mathrm{~cm}^{-3}$, ou seja, solos menos compactados. Nesse sentido, apesar de não ter sido verificada diferença estatística entre os tratamentos, os 
valores encontrados para o preparo do solo com enxada rotativa e plantio em cova pequena aproximaram-se da densidade ideal recomendada para a nogueira-pecã.

Para as propriedades químicas, cabe ressaltar os baixos valores de $\mathrm{pH}$ e $\mathrm{Zn}$ encontrados no solo em todas as áreas dos tratamentos. Assim, mesmo que houvessem níveis adequados de $\mathrm{Zn}$, a sua disponibilidade depende do pH do solo, e da aplicação de N e P (Wells, 2013). Seguindo dentro dessa análise, o mesmo autor enfatiza que o P é importante no armazenamento de energia, para produção de madeira e nozes em nogueira-pecã. De acordo com Santos et al. (2008), a dinâmica do fósforo no solo está relacionada a fatores ambientais que controlam a atividade dos microrganismos que, por sua vez, imobilizam ou liberam os íons ortofosfato.

Além de apresentar correlação com a argila, $D$. abderus apresentou correlação significativa positiva entre a abundância e o $\mathrm{pH}$ do solo e significativa negativa entre a abundância e Al. Em estudo realizado por Portilho et al. (2011), com avaliação da fauna edáfica em sistemas de integração lavoura-pecuária, foi observada correlação significativa positiva entre $\mathrm{Al}$ e coleópteros da Família Chrysomelidae. Segundo os autores, a família em questão pode atuar como indicador sensível de ambientes em solos com saturação por alumínio. Nesse sentido, no presente trabalho, em todas as áreas avaliadas encontraram-se baixos teores de Al, bem como baixa riqueza de espécies de Chrysomelidae.

Os resultados da mensuração de plantas de nogueirapecã corroboram com estudo desenvolvido na mesma área por Paulus (2015). Segundo o autor, o preparo do solo em cova grande proporcionou maior crescimento em altura e diâmetro, assim como no comprimento de raízes aos 21 meses até os 24 meses após o plantio, enquanto que o plantio em cova pequena resultou em menor crescimento radicular e da parte aérea. Cabe ressaltar, que o incremento apresentado no presente estudo refere-se somente a um ano de avaliação. Averiguações a longo prazo podem vir a elucidar melhor os resultados encontrados.

Verifica-se, a partir dos resultados de crescimento das plantas de nogueira-pecã, que o tratamento com plantio em cova grande apresenta maior crescimento em diâmetro e mantém o equilíbrio ambiental existente nas áreas em que foi implantado. Esse fato é corroborado pelos parâmetros ecológicos observados para a área do tratamento $\mathrm{Cg}$, em detrimento dos demais tratamentos, uma vez que apresentou bons índices de diversidade e uniformidade para as espécies de Coleoptera, bem como a riqueza observada nas condições relatadas no presente trabalho.

Nesse sentido, Santana et al. (2013) realizaram um levantamento da fauna epiedáfica na mesma área deste estudo, no primeiro semestre de 2012, ou seja, logo após o preparo do solo e plantio das mudas de nogueira-pecã. Os autores chegaram à conclusão que a manutenção de um ambiente sem perturbação favorece a diversidade e abundância de organismos, uma vez que o tipo de preparo de solo interfere na comunidade de solo.

\section{Conclusão}

O plantio de Carya illinoinensis em Argissolo Vermelho, realizado em cova grande de $40 \mathrm{~cm}$ de diâmetro e $60 \mathrm{~cm}$ de profundidade, nas condições do presente estudo, não interfere na diversidade de coleópteros e promove o maior crescimento em diâmetro de plantas de nogueira-pecã.

\section{Agradecimentos}

À Coordenação de Aperfeiçoamento de Pessoal de Nível Superior (CAPES), pela concessão de bolsa ao primeiro autor. À Fundação Estadual de Pesquisa Agropecuária (FEPAGRO Florestas), pela disponibilidade da área do estudo. À Divinut - Indústria de Nozes, pela doação das mudas de nogueira-pecã.

\section{Referências}

Alvares, C. A. et al. Köppen's climate classification map for Brazil. Meteorologische Zeitschrift, v. 22, n. 6, p. 711-728, 2013. DOI: 10.1127/0941-2948/2013/0507.

Aquino, A. M. et al. Recomendação para coleta de artrópodes terrestres por armadilhas de queda (pit-fall traps). Seropédica: Embrapa Agrobiologia, 2006. 8 p. (Embrapa Agrobiologia. Circular técnica, 18).

Baker, J. B. \& Broadfoot, W. M. A practical field method of site evaluation for eight important southern hardwoods. New Orleans: USDA Forest Service, Southern Forest Experiment Station, 1979. $31 \mathrm{p}$.

Baretta, D. et al. Efeito do cultivo do solo sobre a diversidade da fauna edáfica no planalto sul catarinense. Revista de Ciências Agroveterinárias, v. 5, n. 2, p. 108-117, 2006.

Baretta, D. et al. Fauna edáfica e qualidade do solo. Tópicos em Ciência do Solo, v. 7, p. 119-170, 2011. 
Bignell, D. et al. Macrofauna. In: Moreira, F. M. S. et al. (Ed.). Manual de biologia dos solos tropicais: amostragem e caracterização da biodiversidade. Lavras: UFLA, 2010. p. 79-128.

Cajaiba, R. L. \& Silva, W. B. Abundância e diversidade de Coleoptera (Arthropoda: Insecta) de solo em fragmentos de capoeira ao entorno da Zona Urbana do Município de Uruará-PA, Brasil. EntomoBrasilis, v. 8 , n. 1, p. 30-37, 2015. DOI:10.12741/ebrasilis.v8i1.414.

Correia, M. E. F. \& Oliveira, L. C. M. de. Importância da fauna de solo para a ciclagem de nutrientes. In: Aquino, A. M. de \& Assis, R. L. de (Ed.). Processos biológicos no sistema solo-planta: ferramentas para uma agricultura sustentável. Brasília, DF: Embrapa Agrobiologia, 2005. p. 77-99.

Fazolin, M. Análise faunística de insetos coletados em seringueira no Acre. 1991. 236 f. Tese (Doutorado em Engenharia Florestal) Escola Superior de Agricultura Luiz de Queiroz, Universidade de São Paulo, Piracicaba.

Forti, L. C. et al. Ordem Coleoptera. In: Fujihara, R. T. et al. Insetos de importância econômica: guia ilustrado para identificação de famílias. Botucatu: Ed. da FEPAF, 2011. p. 187-226.

Fronza, D. et al. O cultivo de nogueira-pecã. Santa Maria, RS: UFSM, Colégio Politécnico, Núcleo de Fruticultura Irrigada, 2013. $301 \mathrm{p}$.

Garlet, J. et al. Danos provocados por coró-das-pastagens em plantas de eucalipto. Ciência Rural, v. 39, n. 2, p. 575-576, 2009. DOI: 10.1590/S0103-84782008005000079.

Garlet, J. et al. Fauna de Coleoptera edáfica em eucalipto sob diferentes sistemas de controle químico da matocompetição. Floresta e Ambiente, v. 22, n. 2, p. 239-248, 2015. DOI: 10.1590/21798087.078214 .

Lewinsohn, T. M. et al. Conservação de invertebrados terrestres e seus habitats no Brasil. Megadiversidade, v. 1, n. 1, p. 62-69, 2005.

Lima, S. S. et al. Relação entre macrofauna edáfica e atributos químicos do solo em diferentes agroecossistemas. Pesquisa Agropecuária Brasileira, v. 45, n. 3, p. 322-331, 2010. DOI: 10.1590/S0100-204X2010000300013.

Marques, D. M. et al. Macrofauna edáfica em diferentes coberturas vegetais. Bioscience Journal, v. 30, n. 5, p. 1588-1597, 2014.

Marinoni, R. C. et al. Hábitos alimentares em Coleoptera (Insecta). Ribeirão Preto: Holos, 2001. 64 p.
Nunes, L. A. P. L. et al. Diversidade da fauna edáfica em solos submetidos a diferentes sistemas de manejo no semi-árido nordestino. Scientia Agraria, v. 10, n. 1, p. 43-49, 2009.

Paulus, E. Preparo do solo no estabelecimento inicial da nogueirapecã Carya illinoinensis (Wangenh.) K. Koch em Argissolo Vermelho no Rio Grande Do Sul. 2015. 93 f. Tese (Doutorado em Engenharia Florestal) - Universidade Federal de Santa Maria, Santa Maria, RS.

Portilho, I. I. R. et al. Fauna invertebrada e atributos físicos e químicos do solo em sistemas de integração lavoura-pecuária. Pesquisa Agropecuária Brasileira, v. 46, n. 10, p.1310-1320, 2011. DOI: 10.1590/S0100-204X2011001000027.

Raseira, A. A cultura da nogueira-pecã (Carya illinoensis). Pelotas: EMBRAPA-CNPFT, 1990. 3 p. (EMBRAPA-CNPFT. Comunicado técnico, 63).

Salvadori, J. R. \& Pereira, P. R. V. S. Manejo integrado de corós em trigo e culturas associadas. Passo Fundo: Embrapa Trigo, 2006. 9 p. (Embrapa Trigo. Comunicado técnico online, 203).

Santana, N. A. et al. Efeito do preparo do solo na fauna epiedáfica em cultivo de nogueira-pecã. Cadernos de Agroecologia, v. 8, n. 2, p. 1-5, 2013.

Santos, D. R. et al. Fatores que afetam a disponibilidade do fósforo e o manejo da adubação fosfatada em solos sob sistema plantio direto. Ciência Rural, v. 38, n. 2, p. 576-586, 2008. DOI: 10.1590/ S0103-84782008000200049.

Silva, M. T. B. \& Salvadori, J. R. Coró-das-pastagens. In: Salvadori, J. R. et al. Pragas de solo no Brasil. Passo Fundo: Embrapa Trigo, 2004. v. 1. p. 191-210.

Silva, P. G. da \& Silva, F. C. G. da. Besouros (Insecta: Coleoptera) utilizados como bioindicadores. Revista Congrega Urcamp, v. 5, p. 1-16, 2011.

Sociedade Brasileira de Ciência do Solo. Comissão de Química e Fertilidade do Solo. Manual de adubação e de calagem para os Estados do Rio Grande do Sul e de Santa Catarina. 10. ed. Porto Alegre, 2004. $400 \mathrm{p}$.

Streck, E. V. et al. Solos do Rio Grande do Sul. 2. ed. Porto Alegre: Emater-RS/ASCAR, 2008. 222 p.

Townsend, C. R. et al. Fundamentos em ecologia. 2. ed. Porto Alegre: Armed, 2006. 592 p.

Wells, L. Southeastern pecan growers' handbook. Georgia: University of Georgia, 2013. 236 p. 